\title{
REMOÇÃO DE CROMO (VI) DE SOLUÇÕES AQUOSAS UTILIZANDO O COMPÓSITO MAGNÉTICO CALCINADO HIDROTALCITA-ÓXIDO DE FERRO: ESTUDO CINÉTICO E DE EQUILÍBRIO TERMODINÂMICO
}

\author{
Thiago Vinícius Toledo \\ Instituto Federal de Educação, Ciência e Tecnologia de Minas Gerais, Campus Governador Valadares, Av. Minas Gerais, 5189, \\ 35057-760 Governador Valadares - MG, Brasil \\ Carlos Roberto Bellato* e Kenia Dias Pessoa \\ Departamento de Química, Universidade Federal de Viçosa, Campus Universitário, 36570-000 Viçosa - MG, Brasil \\ Maurício Paulo Ferreira Fontes \\ Departamento de Solos, Universidade Federal de Viçosa, Campus Universitário, 36570-000 Viçosa - MG, Brasil
}

Recebido em 29/5/12; aceito em 17/9/12; publicado na web em 23/1/13

\begin{abstract}
REMOVAL OF CHROMIUM (VI) FROM AQUEOUS SOLUTIONS USING THE CALCINED MAGNETIC COMPOSITE HYDROTALCITE-IRON OXIDE: KINETIC AND THERMODYNAMIC EQUILIBRIUM STUDIES. In this work, hydrotalcite, a layered double hydroxide, had its ion exchange properties combined with the magnetic properties of iron oxide to produce a magnetic adsorbent, HT-Fe 500. These magnetic composites can be used as adsorbents for anionic contaminants in water and subsequently removed from the medium by a simple magnetic process. Removal of chromium (VI) from aqueous solutions using HT-Fe 500 was achieved using batch adsorption experiments. The adsorption capacity, calculated with the Langmuir-Freundlich model showed to be dependent on temperature, reaching values of 25.93 and $48.31 \mathrm{mg} \mathrm{g}^{-1}$, respectively, for temperatures of 25 and $30{ }^{\circ} \mathrm{C}$.
\end{abstract}

Keywords: chromium (VI); adsorption; hydrotalcite-iron oxide composite.

\section{INTRODUÇÃO}

O cromo é um metal de cor cinza obtido do mineral cromita ( $\mathrm{FeO}$. $\mathrm{Cr}_{2} \mathrm{O}_{3}$ ) e pode ser encontrado em solos, águas, rochas, fauna, flora e até mesmo na poeira vulcânica. Este elemento é usado em indústrias de galvanoplastia, na fabricação de ligas como o aço inoxidável, em indústrias de corantes, de curtimento de couro, no tratamento da madeira, entre outros. ${ }^{1,2}$

No ambiente aquático o cromo existe nos estados trivalente, $\mathrm{Cr}(\mathrm{III})$, e hexavalente, $\mathrm{Cr}(\mathrm{VI})$, e exibe diferentes propriedades químicas que estão relacionadas ao seu estado de oxidação. Enquanto o cromo trivalente é um micronutriente essencial, associado ao metabolismo de lipídios e carboidratos e cuja ausência está relacionada à diabetes e doenças cardiovasculares, o $\mathrm{Cr}(\mathrm{VI})$ é considerado perigoso para a saúde pública devido às suas propriedades mutagênicas e carcinogênicas, sendo $0,05 \mathrm{mg} \mathrm{L}^{-1}$ a concentração máxima permitida em águas destinadas ao consumo humano..$^{3-5} \mathrm{Em}$ solução aquosa, $\mathrm{Cr}(\mathrm{VI})$ existe principalmente nas formas de cromato $\left(\mathrm{CrO}_{4}{ }^{2-}\right)$, dicromato $\left(\mathrm{Cr}_{2} \mathrm{O}_{7}^{2-}\right)$ e cromato de hidrogênio $\left(\mathrm{HCrO}_{4}^{-}\right)$. Em soluções básicas ocorre a predominância da espécie $\mathrm{CrO}_{4}{ }^{2-}$ e em soluções extremamente ácidas com valores de $\mathrm{pH}<1$, a espécie predominante é $\mathrm{H}_{2} \mathrm{CrO}_{4}$. Já para a faixa de $\mathrm{pH}$ compreendida entre 2 e 6 , predominam as espécies $\mathrm{HCrO}_{4}^{-}$e $\mathrm{Cr}_{2} \mathrm{O}_{7}^{2-.6}$

Reações de oxidação e redução podem converter $\mathrm{Cr}(\mathrm{III})$ para $\mathrm{Cr}(\mathrm{VI})$ e vice-versa. Esses processos dependem do $\mathrm{pH}$, da concentração de oxigênio, da presença de redutores apropriados e de mediadores que podem atuar como ligantes ou catalisadores. ${ }^{7}$

Os métodos mais utilizados para remover $\mathrm{Cr}(\mathrm{VI})$ de águas residuárias incluem filtração por membranas, precipitação química, osmose reversa, evaporação, troca iônica, extração por solventes, adsorção, entre outros. Entre estes métodos, a adsorção se destaca como processo físico-químico eficaz para a remoção de íons de metais pesados

*e-mail: bellato@ufv.br de águas até mesmo quando presentes em baixas concentrações. ${ }^{5,8}$

Hidróxidos duplos lamelares (HDLs) são hidróxidos metálicos mistos que têm fórmula geral expressa por $\left[\mathrm{M}_{1-\mathrm{x}}{ }^{2+} \mathrm{M}_{\mathrm{x}}{ }^{3+}(\mathrm{OH})_{2}\right]^{\mathrm{x}+} \mathrm{A}_{\mathrm{x} / \mathrm{n}}{ }^{\mathrm{n}-} \cdot \mathrm{mH}_{2} \mathrm{O}$, onde $\mathrm{M}^{2+}$ e $\mathrm{M}^{3+}$ representam, respectivamente, cátions metálicos di e trivalente, $\mathrm{A}^{\mathrm{n}-}$ um ânion de carga $\mathrm{n}$, $\mathrm{x}$ é a razão entre os cátions di e trivalente e m, o número de moléculas de água. A estrutura cristalina destes compostos consiste em camadas carregadas positivamente $\left[\mathrm{M}^{2+}{ }_{1-\mathrm{x}} \mathrm{M}_{\mathrm{x}}{ }^{3+}(\mathrm{OH})_{2}\right]^{\mathrm{x+}}$, semelhantes à estrutura lamelar da brucita $\left(\mathrm{Mg}(\mathrm{OH})_{2}\right)$, e uma região interlamelar negativa composta de ânions e moléculas de água, $\mathrm{A}_{\mathrm{x} / \mathrm{n}}{ }^{\mathrm{n}-} \cdot \mathrm{mH}_{2} \mathrm{O} .{ }^{9}$ A classe mais importante dos HDLs são as hidrotalcitas, que apresentam a fórmula geral $\left[\mathrm{Mg}^{2+}{ }_{1-\mathrm{x}} \mathrm{Al}_{\mathrm{x}}{ }^{3+}(\mathrm{OH})_{2}\right]^{\mathrm{x}+}\left[\mathrm{A}_{\mathrm{x} / \mathrm{n}}{ }^{\mathrm{n}-} \cdot \mathrm{mH}_{2} \mathrm{O}\right]^{\mathrm{x}-}$, onde $\mathrm{x}$ pode ter $\mathrm{o}$ valor entre 0,20 e 0,33 . Os ânions $\left(\mathrm{A}^{\mathrm{n}-}\right)$ e as moléculas de água interlamelares podem ser trocados por outros ânions, o que, em teoria, faz das hidrotalcitas bons trocadores aniônicos. ${ }^{10}$

Nos últimos anos muitos estudos têm sido dedicados à investigação da habilidade dos hidróxidos duplos lamelares em remover oxiânions nocivos, tais como arsenato, cromato etc., de águas contaminadas, por meio da adsorção em superfície e da troca aniônica destes pelos ânions interlamelares da estrutura do HDL. ${ }^{11}$ No entanto, após o processo de adsorção torna-se difícil a remoção destes adsorventes do meio aquoso até mesmo com o auxílio de alguns métodos tradicionais de separação, tais como filtração e sedimentação. ${ }^{12}$ Desta forma, a tecnologia magnética é uma solução promissora. Materiais inorgânicos ou poliméricos têm sido utilizados como suporte para obtenção de adsorventes magnéticos, que são facilmente removidos da solução aquosa através da aplicação de um campo magnético, o que diminui, naturalmente, o custo e o tempo gastos na recuperação do material adsorvedor. ${ }^{13}$

Neste trabalho, as propriedades de troca aniônica e adsorção da hidrotalcita foram combinadas com as propriedades magnéticas do óxido de ferro para produzir um adsorvente magnético, a hidrotalcita-óxido de ferro (HT-Fe). Após calcinação do HT-Fe a $500{ }^{\circ} \mathrm{C}$ por 4 h, obteve-se o compósito HT-Fe 500, que teve sua eficiência avaliada 
na remoção de $\mathrm{Cr}(\mathrm{VI})$ de soluções aquosas. Para isso, verificou-se o efeito da temperatura $\left(25\right.$ a $\left.45^{\circ} \mathrm{C}\right)$ nas isotermas de equilíbrio de adsorção, o comportamento termodinâmico de adsorção através das variações de entalpia, entropia e energia livre de Gibbs e o ajuste de modelos cinéticos capazes de fornecer informações sobre o mecanismo do processo adsortivo.

\section{PARTE EXPERIMENTAL}

\section{Síntese da hidrotalcita (HT) e da hidrotalcita-óxido ferro calcinada (HT-Fe 500)}

A síntese da hidrotalcita foi realizada pelo método de coprecipitação a $\mathrm{pH}$ variável, conforme descrito na literatura, utilizando a razão molar $\mathrm{Al} /(\mathrm{Al}+\mathrm{Mg})$ igual a 0,33 , em presença de $\mathrm{NaOH}$ e $\mathrm{Na}_{2} \mathrm{CO}_{3} \cdot{ }^{14}$ Desta forma, $100 \mathrm{~mL}$ de uma solução $1,01 \mathrm{~mol} \mathrm{~L}^{-1}$ de $\mathrm{Mg}\left(\mathrm{NO}_{3}\right)_{2} \cdot 6 \mathrm{H}_{2} \mathrm{O}$ e $0,510 \mathrm{~mol} \mathrm{~L}^{-1} \mathrm{de} \mathrm{Al}\left(\mathrm{NO}_{3}\right)_{3} \cdot 9 \mathrm{H}_{2} \mathrm{O}$ foram adicionados $\left(1 \mathrm{~mL} \mathrm{~min}{ }^{-1}\right)$, com o auxílio de uma bomba peristáltica, a $100 \mathrm{~mL}$ de uma solução básica de $\mathrm{NaOH} 3,61 \mathrm{~mol} \mathrm{~L}^{-1}$ e $\mathrm{Na}_{2} \mathrm{CO}_{3}$ 1,68 mol L-1, mantida sob vigorosa agitação e à temperatura ambiente. A mistura reacional foi mantida sob agitação por mais 140 min e o gel formado foi seco em estufa por $18 \mathrm{~h} \mathrm{a} 60^{\circ} \mathrm{C}$. Posteriormente, lavou-se o material com água deionizada até que o $\mathrm{pH}$ da água de lavagem adquirisse a neutralidade. Em seguida, o material sólido obtido - hidrotalcita (HT) - foi seco em estufa a $80{ }^{\circ} \mathrm{C}$ por mais $12 \mathrm{~h}$, macerado e acondicionado em dessecador. ${ }^{9}$

Na síntese do compósito magnético HT-Fe ajustou-se uma quantidade da hidrotalcita obtida a uma razão em massa de HT:óxido de ferro de 1:0,3. Esta é a menor razão na qual o compósito ainda manifestou propriedades magnéticas e que resultou em menor consumo de reagentes e um ganho na capacidade de adsorção do adsorvente, pois o óxido de ferro na superfície pode bloquear parte dos sítios de adsorção do HDL de partida. ${ }^{9,15}$ Desta forma, prepararam-se $400 \mathrm{~mL}$ de uma suspensão de $\mathrm{HT}$, com $\mathrm{FeCl}_{3} \cdot 6 \mathrm{H}_{2} \mathrm{O}$ e $\mathrm{FeSO}_{4} .7 \mathrm{H}_{2} \mathrm{O}$ nas proporções de 1,$25 ; 0,25$ e $0,6 \mathrm{~g} \mathrm{~L}^{-1}$, respectivamente. A suspensão foi aquecida até $70{ }^{\circ} \mathrm{C}$ e a precipitação do óxido de ferro foi obtida pela adição de $30 \mathrm{~mL}$ de $\mathrm{NaOH} 5 \mathrm{~mol} \mathrm{~L}^{-1}$. O material obtido (HT-Fe), após lavado com água deionizada, foi seco em estufa a $70{ }^{\circ} \mathrm{C}$ por $18 \mathrm{~h}$.

Para a obtenção do compósito calcinado (HT-Fe 500), o HT-Fe foi mantido em mufla por $4 \mathrm{~h}$ a $500{ }^{\circ} \mathrm{C}$ e acondicionado em dessecador para uso posterior.

\section{Caracterização dos materiais}

Os materiais preparados foram caracterizados por técnicas complementares: difratometria de raios- $\mathrm{X}$, microscopia eletrônica de varredura e espectroscopia no infravermelho. As análises de difração de raios-X foram realizadas em difratômetro X-ray Diffraction System modelo X' Pert PRO (PANalytical) utilizando filtro de Ni e radiação Co-k $\alpha(\lambda=1,78890 \AA)$ e variação angular de $10-80^{\circ}(2 \theta)$. Para as análises de microscopia eletrônica de varredura as amostras que não são condutoras de corrente elétrica devem ser previamente metalizadas com um material condutor. Desta forma, as amostras foram previamente recobertas, através da precipitação a vácuo, com uma película micrométrica de ouro em metalizador (Sputter coating attachment), modelo SCA 010 e analisadas, em seguida, em um microscópio marca LEO, modelo 1430 VP. O recobrimento seguiu rigorosamente o contorno do material para não resultar em imagens não verdadeiras da topografia da amostra. A análise de espectroscopia no infravermelho foi realizada diretamente sobre a amostra em um espectrofotômetro infravermelho Varian 660-IR com acessório de refletância atenuada Pike GladiATR na região de 400 a $4000 \mathrm{~cm}^{-1}$.

\section{Experimentos de adsorção}

Com o objetivo de avaliar o efeito do $\mathrm{pH}$ sobre a capacidade de adsorção de Cr(VI) pelo compósito HT-Fe 500, foram realizados estudos em batelada para valores inteiros de $\mathrm{pH}$ compreendidos entre 1 e 10. Assim, amostras de $100 \mathrm{mg}$ de HT-Fe 500 foram adicionadas a $20 \mathrm{~mL}$ de soluções de $\mathrm{Cr}(\mathrm{VI})$ a $75 \mathrm{mg} \mathrm{L}^{-1}$ com os diferentes valores de $\mathrm{pH}$ previamente ajustados com soluções diluídas de $\mathrm{NaOH}$ e/ou $\mathrm{HCl}$. O sistema permaneceu sob agitação constante a $25^{\circ} \mathrm{C}$ em banho termostatizado por $24 \mathrm{~h}$.

Para os estudos das isotermas de equilíbrio a dosagem do adsorvente em solução foi mantida em 5,0 $\mathrm{g} \mathrm{L}^{-1}$. Nestes estudos, amostras de HT-Fe 500 foram mantidas em contato por $24 \mathrm{~h}$ em banho termostatizado com soluções de $\mathrm{Cr}(\mathrm{VI})$ em diferentes concentrações (10 a $200 \mathrm{mg} \mathrm{L}^{-1}$ ) e valores de $\mathrm{pH}$ previamente ajustados para 7. A fim de avaliar o efeito da temperatura no processo de adsorção, as isotermas foram realizadas a $25,30,35,40$ e $45^{\circ} \mathrm{C}$.

A análise da cinética do processo de adsorção foi realizada em batelada a $25^{\circ} \mathrm{C}$, onde a dosagem do HT-Fe 500 em solução foi mantida a 5,0 $\mathrm{g} \mathrm{L}^{-1}$ em soluções de $\mathrm{Cr}(\mathrm{VI})$ de diferentes concentrações $\left(75,100\right.$ e $\left.200 \mathrm{mg} \mathrm{L}^{-1}\right)$ com valores de $\mathrm{pH}$ previamente ajustados para 7,0. As amostras foram agitadas e alíquotas do sobrenadante retiradas em diferentes períodos de tempo (30, 60, 90, 120, 150, 180, 210, 240, 270, 300, 330, 360, 390, 420 e $450 \mathrm{~min})$.

Em todos os estudos, os ensaios foram realizados em duplicata e a concentração de $\mathrm{Cr}(\mathrm{VI})$ foi determinada por espectroscopia de absorção atômica (EAA). A quantidade de $\mathrm{Cr}$ (VI) por unidade de massa do adsorvente (q) dada em $\mathrm{mg} \mathrm{g}^{-1}$ foi calculada a partir da Equação 1:

$$
\mathrm{q}=\left(\frac{\mathrm{C}_{0}-\mathrm{Ce}}{\mathrm{M}}\right) \times \mathrm{V}
$$

onde $\mathrm{C}_{0}$ e Ce $\left(\mathrm{mg} \mathrm{L}^{-1}\right)$ são, respectivamente, as concentrações inicial e de equilíbrio de $\mathrm{Cr}(\mathrm{VI}), \mathrm{M}$ é a massa de HT-Fe 500 (g) e V o volume da solução (L).

A solução estoque de $\mathrm{Cr}(\mathrm{VI}), 1000 \mathrm{mg} \mathrm{L}^{-1}$, foi preparada pela dissolução de 2,8290 g de $\mathrm{K}_{2} \mathrm{Cr}_{2} \mathrm{O}_{7}$, previamente seco em mufla, em água deionizada utilizando um balão volumétrico de $1000 \mathrm{~mL}$. As soluções de diferentes concentrações de $\mathrm{Cr}(\mathrm{VI})$ usadas nos experimentos foram obtidas a partir de diluições da solução estoque.

\section{RESULTADOS E DISCUSSÃO}

\section{Caracterização dos materiais}

Os resultados da análise por difratometria de raios-X confirmaram que a hidrotalcita foi devidamente sintetizada, apresentando um padrão de difração de acordo com os padrões cristalográficos da literatura, ou seja, os picos obtidos para os valores de $2 \theta$ iguais a $13,70^{\circ}$ e $27,4^{\circ}$ correspondem, respectivamente, aos planos de difração em $\left(\begin{array}{lll}0 & 0 & 3\end{array}\right)$ e $\left(\begin{array}{lll}0 & 0 & 6\end{array}\right)$, o que indica que o material sintetizado apresenta simetria romboédrica. ${ }^{16,17}$ A definição dos picos encontrados indicou ainda uma alta cristalinidade para a mesma, o que se deve à proximidade dos raios iônicos de $\mathrm{Mg}$ e $\mathrm{Al}(0,66$ e $0,51 \AA$ A , respectivamente) ${ }^{17}$ Os difratogramas da hidrotalcita (HT), da hidrotalcita-óxido de ferro (HT-Fe), da hidrotalcita-óxido de ferro calcinada a $500{ }^{\circ} \mathrm{C}$ (HT-Fe 500) e da hidrotalcita reidratada em solução de Cr(VI), HT-Fe 500-Cr(VI) são mostrados na Figura 1. Observa-se na Figura 1 que não houve alteração estrutural da hidrotalcita devido à incorporação do óxido de ferro, uma vez que grande parte dos picos está sobreposta, indicando que ocorreu apenas a deposição do óxido de ferro em sua superfície. A calcinação destruiu a estrutura lamelar do compósito HT-Fe, transformando-o em um óxido misto, o que é evidenciado pela 
ausência dos picos em $13,7^{\circ}$ e $27,4^{\circ}$ no difratograma do HT-Fe 500. Já pelo difratograma do compósito HT-Fe 500-Cr(VI) ficou evidenciado o efeito memória (restabelecimento da estrutura lamelar) deste HDL, uma vez que os picos correspondentes ao compósito original foram restabelecidos após o contato do adsorvente com a solução do contaminante cromo hexavalente. No entanto, houve uma diminuição da intensidade destes picos restabelecidos e, consequentemente, uma diminuição do espaçamento interlamelar, fato que se deve à substituição dos íons carbonato, da região interlamelar, pelas espécies aniônicas de $\mathrm{Cr}(\mathrm{VI})$, pois o raio iônico do $\mathrm{Cr}^{6+}$ é aproximadamente quatro vezes menor que o raio iônico do $\mathrm{CO}_{3}{ }^{2-} \cdot{ }^{18,19}$

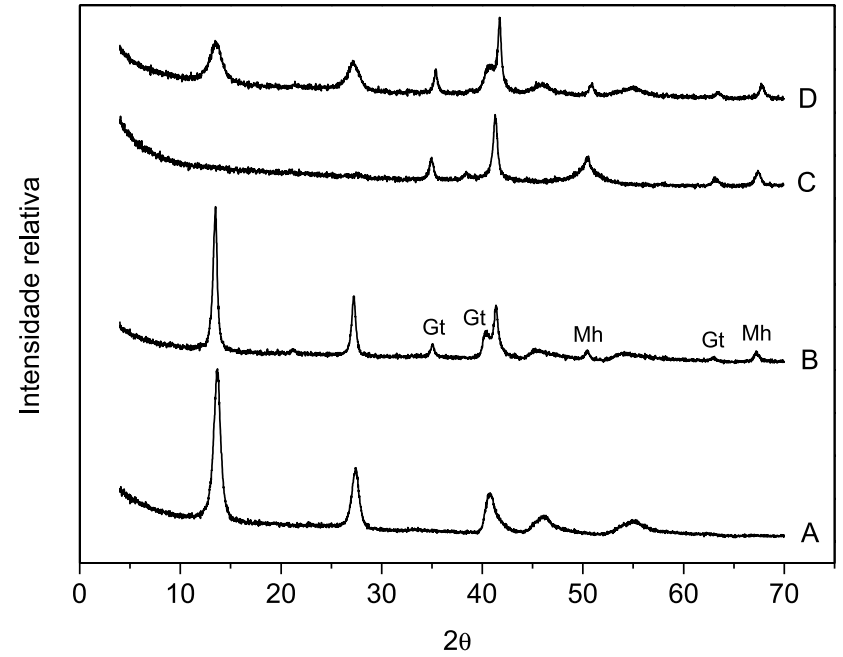

Figura 1. Difratogramas de raios-X: hidrotalcita, $H T(A)$, hidrotalcita-óxido de ferro $(\mathrm{HT}-\mathrm{Fe})(\mathrm{B})$, hidrotalcita-óxido de ferro calcinada a $500{ }^{\circ} \mathrm{C}(\mathrm{HT}-\mathrm{Fe}$ 500) (C) e hidrotalcita-óxido de ferro reidratado em solução de $\mathrm{Cr}(\mathrm{VI})$ (HT-Fe $500-C r(V I))(D) .(G t=$ goethita e $M h=$ maghemita $)$

Devido às condições de síntese, o óxido de ferro presente na superfície da hidrotalcita apresenta-se como uma mistura de óxidos. Pela análise de difratometria de raios-X (Figura 1) pode-se observar a mistura de óxidos, formada pela presença de $\alpha-\mathrm{FeO}(\mathrm{OH})$ (goethita), evidenciada pelos picos em $\mathrm{d}=0,4193 ; 0,2098$ e $0,1613 \mathrm{~nm}$ e $\gamma-\mathrm{Fe}_{2} \mathrm{O}_{3}$ (maghemita) em d = 0,2506 e 0,2956 nm..$^{20}$

A maghemita, presente na superfície do adsorvente sintetizado conferiu a ele propriedades magnéticas, conforme mostrado na Figura 1S (material suplementar). As propriedades magnéticas do material representam grande vantagem porque permitem que o adsorvente seja facilmente removido da solução aquosa, após adsorção do contaminante, por um simples processo magnético.

A microscopia eletrônica de varredura (MEV) foi utilizada para avaliar a forma e a topografia da HT, do compósito magnético HT-Fe, do compósito HT-Fe 500 e do compósito HT-Fe reidratado em solução de $\mathrm{Cr}(\mathrm{VI})$. As micrografias de MEV para todos estes compósitos são apresentadas na Figura 2.

A partir da Figura 2A é possível visualizar que a hidrotalcita é composta por lâminas, que se referem à sobreposição das camadas dos octaedros da estrutura lamelar, cujos vértices são ocupados por hidroxilas e os centros, de forma alternada, pelos cátions metálicos $\mathrm{Mg}^{2+}$ e $\mathrm{Al}^{3+}$. A Figura 2B mostra que na superfície da hidrotalcita ocorreu a deposição de óxido de ferro, formando um aglomerado de aparência esponjosa e com cristais de pequeno tamanho, que é responsável pelo aumento da heterogeneidade da superfície deste adsorvente. ${ }^{21}$ Já com a calcinação (Figura 2C), observou-se a compactação do adsorvente, onde há um maior preenchimento dos espaços e, consequentemente, uma melhor distribuição do óxido de
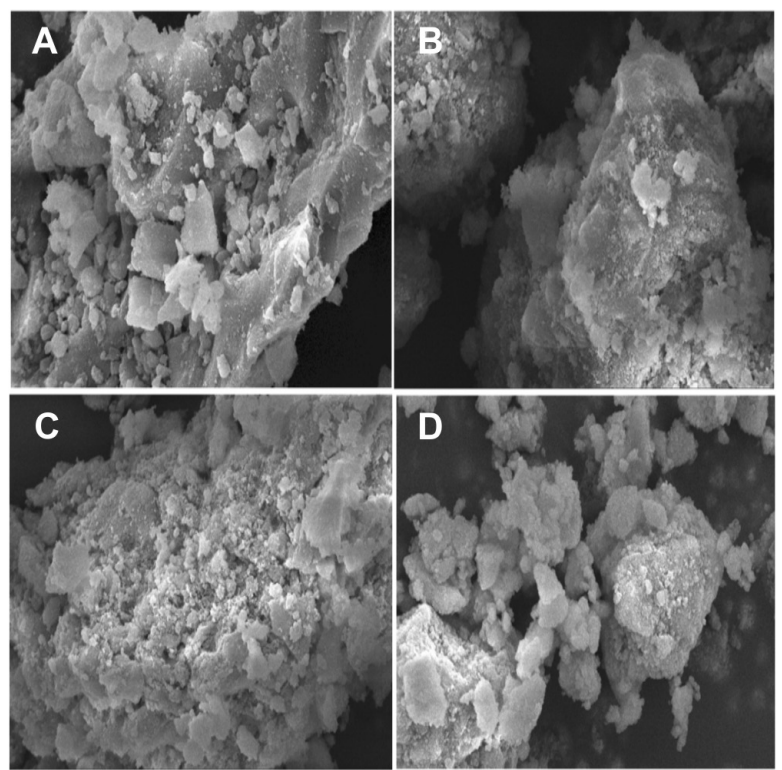

Figura 2. Micrografias com escala de $10 \mu \mathrm{m}$ e ampliação de 4.000 vezes da HT (A), do compósito HT-Fe (B), do compósito HT-Fe $500(C)$ e do compósito HT-Fe 500-Cr(VI) (D)

ferro pela superfície da hidrotalcita. Isto ocorre devido à eliminação da água e do ânion interlamelar carbonato na forma de $\mathrm{CO}_{2}$, o que faz com que a estrutura lamelar entre em colapso e se desestabilize. $\mathrm{Na}$ Figura 2D evidencia-se a recuperação da aparência esponjosa menos uniforme, semelhante à apresentada na Figura 2B, fato este que se deve à reconstrução da estrutura lamelar (efeito memória) para o compósito, obtida pela incorporação de moléculas de água e espécies do contaminante $\mathrm{Cr}(\mathrm{VI})$.

Na Figura 3 são mostrados os espectros obtidos na região do infravermelho para o compósito HT-Fe 500 (A), para o HT-Fe (B) e (C) para o compósito HT-Fe 500-Cr(VI). Na Figura 3A não foram evidenciadas bandas de ligações características da molécula de água e do ânion carbonato, o que confirma a eliminação destes pela calcinação realizada a $500{ }^{\circ} \mathrm{C}$. Na Figura $3 \mathrm{~B}$ e C, as bandas de absorção, presentes $\mathrm{a} \sim 3500 \mathrm{~cm}^{-1}$, são atribuídas às vibrações de estiramento da ligação $\mathrm{H}-\mathrm{O}$ dos grupos $\mathrm{OH}$ da camada de hidróxidos; uma banda referente à vibração da água aparece em $1600 \mathrm{~cm}^{-1}$ e sua

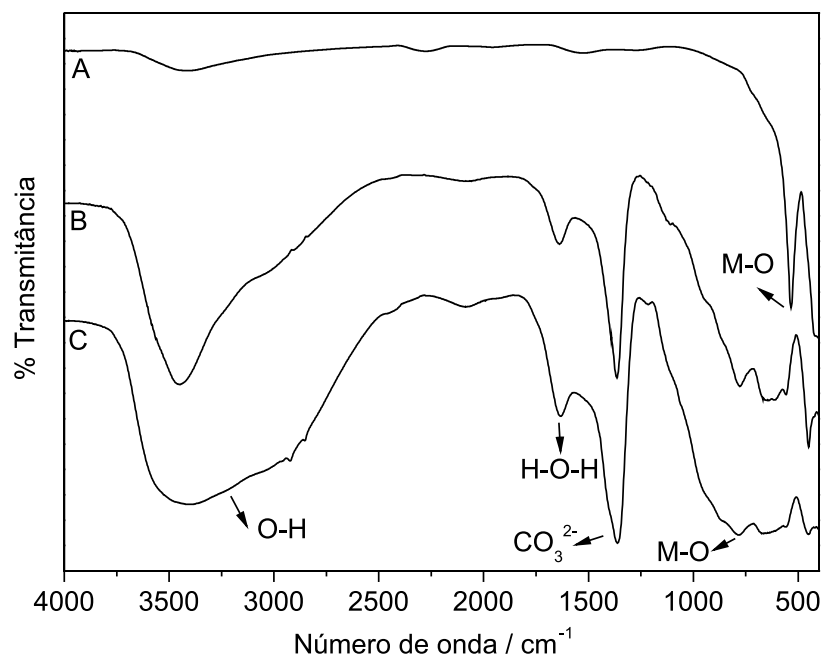

Figura 3. Espectros de infravermelho para HT-Fe 500 (A), HT-Fe (B) e HT-Fe 500-Cr(VI) (C) 
intensidade é dependente do tipo de ânion e da quantidade de água na região interlamelar. ${ }^{22,23}$

O ânion carbonato em um ambiente simétrico é caracterizado por uma simetria planar $\mathrm{D} 3 \mathrm{~h}$, com três bandas de absorções ativas no infravermelho, como no caso do ânion carbonato livre. No espectro do HT-Fe estas bandas ocorreram entre 450 a $780 \mathrm{~cm}^{-1}$, onde também foram evidenciadas as bandas correspondentes às vibrações das ligações Al-O e Mg-O. A presença de um ombro em torno de 1400 $\mathrm{cm}^{-1}$ ou de uma banda na região de $1360 \mathrm{~cm}^{-1}$ tem sido atribuída à redução da simetria do carbonato (sítio de simetria $\mathrm{C} 2 \mathrm{v}$ ) e à desordem natural no espaço interlamelar. ${ }^{24}$ Observou-se ainda para o HT-Fe um deslocamento das bandas, que pode ser atribuído a uma perturbação do ânion carbonato com o óxido de ferro, cuja ligação $\mathrm{Fe}-\mathrm{O}$ se manifesta na banda evidenciada em $\sim 602 \mathrm{~cm}^{-1}$. $^{23} \mathrm{~A}$ semelhança observada para os espectros de infravermelho B e C comprova a eficiência da reconstrução estrutural do HDL original após a sua reidratação e incorporação do cromo hexavalente.

\section{Efeito do pH inicial}

A Figura 4 mostra a eficiência na remoção de Cr(VI) pelo compósito calcinado HT-Fe 500 para os diferentes valores de $\mathrm{pH}$ avaliados.

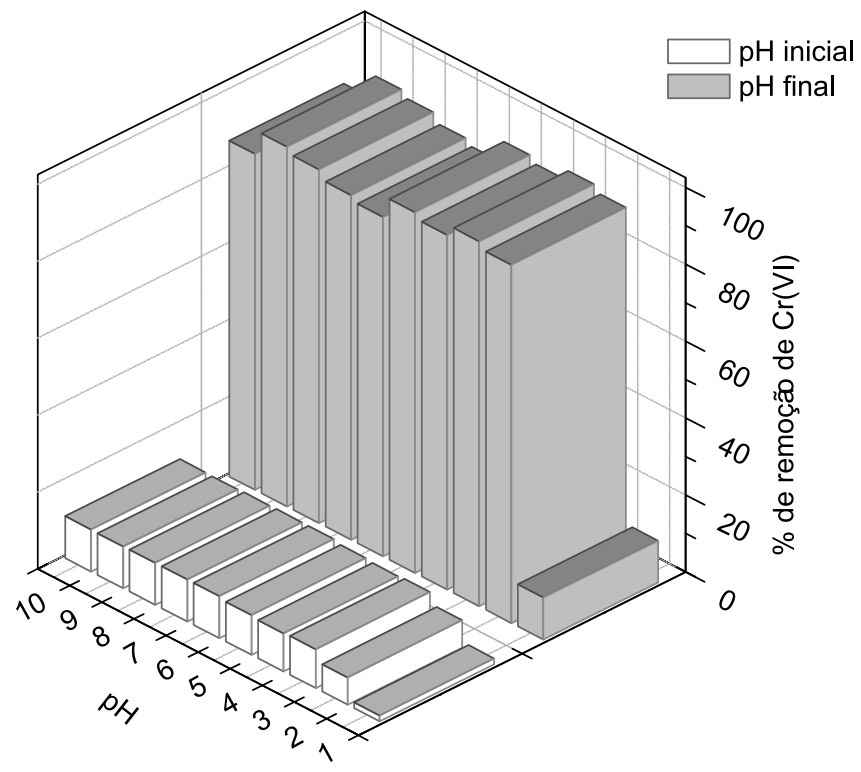

Figura 4. Avaliação do pH na adsorção de Cr(VI) pelo compósito HT-Fe 500

A Figura 4 mostra que, em valor de $\mathrm{pH}$ inicial da solução de cromo hexavalente igual a 1,0, a eficiência de remoção foi apenas de $10,9 \%$. Já para os valores de $\mathrm{pH}$ compreendidos entre 2,0 a 10,0 houve um aumento considerável e similar na quantidade de $\mathrm{Cr}(\mathrm{VI})$ removida, onde o menor valor obtido foi de $87,4 \%$ em valor de $\mathrm{pH}$ inicial da solução igual a 10,0 e a maior remoção percentual $(94,9 \%)$ foi encontrada para $\mathrm{pH}$ inicial da solução igual a 3,0. O contato entre adsorvente e as soluções do contaminante proporcionou ainda um aumento nos valores de $\mathrm{pH}$ inicial destas para valores maiores que 9,8, exceto para a solução de $\mathrm{pH}$ inicial igual a 1,0 que teve seu valor de $\mathrm{pH}$ final alterado para 1,5. O aumento do $\mathrm{pH}$ da solução se deve ao processo envolvido na adsorção de $\mathrm{Cr}(\mathrm{VI})$ que, no caso de um material calcinado, ocorre através da reidratação do adsorvente, capaz de intercalar espécies do oxiânion contaminante na região interlamelar e tornar as soluções alcalinas, pois parte das hidroxilas $\left(\mathrm{OH}^{-}\right)$do $\mathrm{HDL}$ original são substituídas pelo oxiânion na reconstrução do HDL e são liberadas para a solução. ${ }^{11,25} \mathrm{O}$ aumento da alcalinidade das soluções favoreceu a remoção do metal, pois a espécie predominante em meio básico é o cromato $\left(\mathrm{CrO}_{4}^{2-}\right)$ que, por ser uma espécie aniônica bivalente, apresenta maior interação eletrostática pela lamela do adsorvente, que tem carga elétrica positiva. Já para a solução onde o pH final foi menor que 2,0, a baixa remoção se deve à predominância da espécie totalmente protonada, $\mathrm{H}_{2} \mathrm{CrO}_{4}$, que por não apresentar carga, apresenta baixa afinidade pelo adsorvente. Outro fator envolvido na baixa eficiência em valores baixos de $\mathrm{pH}$ é a perda de estabilidade dos HDLs e consequente danificação de suas estruturas, o que pode diminuir significativamente a adsorção de oxiânions. ${ }^{6,11} \mathrm{O}$ aumento de $\mathrm{pH}$ da solução após a adsorção de $\mathrm{Cr}(\mathrm{VI})$ faz com que este adsorvente se torne bastante interessante, principalmente para o tratamento de efluentes de galvanoplastia. Os efluentes de galvanoplastia são ácidos e após o processo de adsorção necessitam ser neutralizados para a sua reutilização ou descarte. Como o HT-Fe 500 promove um aumento no valor final de $\mathrm{pH}$ da solução de $\mathrm{Cr}(\mathrm{VI})$, isto representaria uma economia no consumo de reagentes necessários para a realização da neutralização dos efluentes.

Como não houve diferença significativa na eficiência de remoção na faixa de $\mathrm{pH}$ 2,0 a 10,0, os experimentos posteriores foram realizados com valores de $\mathrm{pH}$ inicial das soluções ajustados para 7,0.

\section{Isotermas de adsorção}

Os dados de equilíbrio de adsorção de $\mathrm{Cr}$ (VI) foram explorados usando os modelos de isoterma de Langmuir, Freundlich e LangmuirFreundlich para determinar a correlação entre a massa da fase sólida e a concentração de equilíbrio na fase aquosa. A adsorção segundo a isoterma de Langmuir é dada pela Equação 2:

$$
\mathrm{q}_{\mathrm{e}}=\frac{\mathrm{q}_{\max } \mathrm{bC}_{\mathrm{e}}}{1+\mathrm{bC}_{\mathrm{e}}}
$$

onde $\mathrm{q}_{\max }\left(\mathrm{mg} \mathrm{g}^{-1}\right)$ e b ( $\left.\mathrm{L} \mathrm{mg}^{-1}\right)$ são as constantes de Langmuir associadas à capacidade e à energia de adsorção, qe a quantidade do íon metálico adsorvido ( $\mathrm{mg} \mathrm{g}^{-1}$ ) e $\mathrm{C}_{\mathrm{e}}$ a concentração de equilíbrio, em $\mathrm{mg} \mathrm{L}^{-1}$.

O modelo de Langmuir assume que a adsorção ocorre em monocamada, não há interação entre os íons adsorvidos e os sítios de adsorção são idênticos. ${ }^{26}$ A forma linearizada da Equação de Langmuir é dada pela Equação 3:

$$
\frac{\mathrm{C}_{\mathrm{e}}}{\mathrm{q}_{\mathrm{e}}}=\frac{1}{\mathrm{q}_{\max } \mathrm{b}}+\frac{\mathrm{C}_{\mathrm{e}}}{\mathrm{q}_{\max }}
$$

O gráfico experimental foi obtido plotando $C_{e} / q_{e}$ versus $C_{e}$ e os valores das constantes de Langmuir $\left(\mathrm{q}_{\max } \mathrm{e} b\right)$, obtidos pelo método da regressão linear, são mostrados na Tabela 1.

A isoterma de Freundlich (Equação 4) é uma equação empírica que assume que o processo de adsorção ocorre em multicamadas em superfícies heterogêneas e a capacidade de adsorção está relacionada com a concentração de $\mathrm{Cr}(\mathrm{VI})$ no equilíbrio. ${ }^{27}$

$$
\mathrm{q}_{\mathrm{e}}=\mathrm{K}_{\mathrm{F}} \mathrm{C}_{\mathrm{e}}^{\mathrm{n}}
$$

onde $\mathrm{q}_{\mathrm{e}}$ e $\mathrm{C}_{\mathrm{e}}$ são, respectivamente, a quantidade adsorvida ( $\mathrm{mg} \mathrm{g}^{-1}$ ) e a concentração do adsorvato no equilíbrio $\left(\mathrm{mg} \mathrm{L}^{-1}\right) ; \mathrm{K}_{\mathrm{F}}$ e $\mathrm{n}$, são as constantes de Freundlich relacionadas à capacidade e intensidade de adsorção, respectivamente.

A forma linearizada da Equação de Freundlich é dada pela Equação 5:

$$
\operatorname{lnq}_{\mathrm{e}}=\frac{1}{\mathrm{n}} \ln \mathrm{C}_{\mathrm{e}}+\ln \mathrm{K}_{\mathrm{F}}
$$


Tabela 1. Parâmetros calculados a partir dos modelos de Langmuir, Freundlich e Langmuir-Freundlich para a adsorção de Cr(VI) pela hidrotalcita-óxido de ferro calcinada (HT-Fe 500)

\begin{tabular}{|c|c|c|c|c|c|}
\hline \multirow{2}{*}{ Modelo de Isoterma } & \multicolumn{5}{|c|}{ Temperatura $(\mathrm{K})$} \\
\hline & 298 & 303 & 308 & 313 & 318 \\
\hline \multicolumn{6}{|l|}{ Langmuir } \\
\hline qmax $\left(\mathrm{mg} \mathrm{g}^{-1}\right)$ & 25,92 & 29,66 & 26,99 & 31,63 & 32,35 \\
\hline $\mathrm{b}\left(\mathrm{L} \mathrm{mg}^{-1}\right)$ & 0,13 & 0,12 & 0,11 & 0,11 & 0,084 \\
\hline $\mathrm{R}_{\mathrm{L}}$ & 0,040 & 0,043 & 0,047 & 0,047 & 0,060 \\
\hline $\mathrm{R}^{2}$ & 0,977 & 0,787 & 0,973 & 0,974 & 0,978 \\
\hline \multicolumn{6}{|l|}{ Freundlich } \\
\hline $\mathrm{k}_{\mathrm{F}}\left(\left(\mathrm{mg} \mathrm{g}^{-1}\right)\left(\mathrm{L} \mathrm{mg}^{-1}\right)^{1 / \mathrm{n}}\right)$ & 3,36 & 4,11 & 0,18 & 3,09 & 2,45 \\
\hline $\mathrm{n}$ & 1,83 & 1,99 & 0,63 & 1,58 & 1,50 \\
\hline $\mathrm{R}^{2}$ & 0,735 & 0,866 & 0,934 & 0,852 & 0,716 \\
\hline \multicolumn{6}{|l|}{ Langmuir-Freundlich } \\
\hline $\mathrm{qmax}\left(\mathrm{mg} \mathrm{g}^{-1}\right)$ & 25,93 & 48,31 & 39,78 & 38,56 & 40,17 \\
\hline $\mathrm{b}\left(\mathrm{L} \mathrm{mg}^{-1}\right)$ & 0,16 & 0,35 & 0,40 & 0,10 & 0,056 \\
\hline $\mathrm{n}$ & 0,16 & 0,75 & 0,77 & 0,93 & 0,91 \\
\hline $\mathrm{R}^{2}$ & 0,989 & 0,997 & 0,993 & 0,996 & 0,996 \\
\hline
\end{tabular}

O gráfico experimental foi obtido plotando $\operatorname{lnq}_{\mathrm{e}}$ versus $\operatorname{lnC}_{\mathrm{e}} \mathrm{e}$ os valores das constantes de Freundlich $\left(\mathrm{K}_{\mathrm{F}}\right.$ e $\left.\mathrm{n}\right)$ foram obtidos pelo método da regressão linear, conforme mostrado na Tabela 1.

O modelo de três parâmetros Langmuir-Freundlich (Equação 6) tem sido aplicado para tratar estudos de adsorção em superfícies heterogêneas. $\mathrm{O}$ limite $\mathrm{n}=1$ corresponde a um sistema homogêneo, enquanto $\mathrm{n}<1$ se refere ao aumento da heterogeneidade. ${ }^{28}$

$$
\mathrm{q}_{\mathrm{e}}=\mathrm{Q} \frac{\left(\mathrm{bC}_{\mathrm{e}}\right)^{1 / \mathrm{n}}}{1+\left(\mathrm{bC}_{\mathrm{e}}\right)^{1 / \mathrm{n}}}
$$

onde $\mathrm{Q}\left(\mathrm{mg} \mathrm{g}^{-1}\right)$ é a quantidade máxima de adsorção que reflete a área ocupada por uma monocamada de adsorvente, $\mathrm{C}_{\mathrm{e}}$ é a concentração de equilíbrio na fase líquida $\left(\mathrm{mg} \mathrm{L}^{-1}\right)$, b é a constante que relaciona a média da afinidade do adsorvato pela superfície do adsorvente ( $\mathrm{L}$ $\mathrm{mg}^{-1}$ ) e $\mathrm{n}$ é o índice de heterogeneidade, que varia de 0 até 1 .

Como mostrado na Tabela 1, os dados de adsorção de $\mathrm{Cr}(\mathrm{VI})$ pelo compósito calcinado HT-Fe 500 se ajustaram melhor ao modelo de isoterma de três parâmetros de Langmuir-Freundlich, uma vez que os coeficientes de correlação $\left(\mathrm{R}^{2}\right)$ foram maiores que os apresentados pelos outros modelos. Este modelo, que é uma combinação dos modelos de Langmuir e Freundlich, também conhecido como modelo Sips, é normalmente utilizado quando se tem sólidos heterogêneos e, de acordo com Brinques, ${ }^{29}$ a relação entre as quantidades de $\mathrm{Cr}(\mathrm{VI})$ livre em solução e adsorvida no HT-Fe 500 é uma forma exponencial. A heterogeneidade da superfície do adsorvente foi confirmada pelos valores de $n<1$. Desta forma, este modelo prediz que para altas concentrações de $\mathrm{Cr}(\mathrm{VI})$ a adsorção ocorre preferencialmente em monocamada na superfície heterogênea do adsorvente..$^{30} \mathrm{O}$ parâmetro $\mathrm{q}_{\max }$ tem sido utilizado para estimar a adsorção máxima e b é a constante relacionada à energia de ligação soluto-superfície do adsorvente. Quanto menores os valores de b, maior a tendência de formação do complexo adsorvente-adsorvato. Desta forma, verifica-se pelos valores de b estimados pelo modelo Sips $(b<0,403)$, que a formação do HT-Fe 500-Cr(VI) é bastante favorável. ${ }^{21}$ Para a faixa de temperatura e concentração estudadas, a capacidade máxima de adsorção prevista pelo modelo Sips esteve entre 25,9 e 48,3 $\mathrm{mg} \mathrm{g}^{-1}$, que é superior a valores estimados pelo modelo de Langmuir para outros hidróxidos duplos lamelares utilizados na adsorção de $\mathrm{Cr}(\mathrm{VI})$, tais como HDL Mg-Al não calcinado $\left(16,3 \mathrm{mg} \mathrm{g}^{-1}\right),{ }^{31} \mathrm{HDL} \mathrm{Mg}-\mathrm{Al}-\mathrm{Zr}$ calcinado a $450{ }^{\circ} \mathrm{C}\left(24 \mathrm{mg} \mathrm{g}^{-1}\right)^{32}$ e HDL $\mathrm{Mg}$-Al carbonato não calcinado $\left(23,2 \mathrm{mg} \mathrm{g}^{-1}\right),{ }^{33}$ o que confirma o bom potencial do HT-Fe 500 na descontaminação de águas contendo espécies de $\mathrm{Cr}(\mathrm{VI})$.

\section{Cinética de adsorção}

As informações sobre a cinética de adsorção são necessárias para selecionar as condições ideais que favorecem a remoção de íons metálicos em processos de batelada. Para investigar o mecanismo de adsorção juntamente com o potencial deste no controle de alguns passos envolvidos no processo, tais como transporte de massa ou reações químicas, os modelos cinéticos têm sido constantemente utilizados para analisar os dados experimentais. ${ }^{34}$ Neste trabalho foram explorados os modelos de pseudoprimeira ordem de Lagergren, pseudossegunda ordem de Ho e difusão intrapartícula.

O modelo de pseudoprimeira ordem de Lagergren ${ }^{35}$ é representado pela seguinte equação:

$$
\log (q e-q t)=\log q_{e}-\frac{K_{1}}{2,303} t
$$

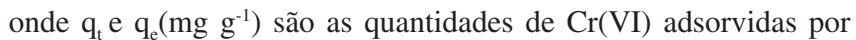
unidade de massa em um tempo t e no equilíbrio, respectivamente, e $\mathrm{K}_{1}\left(\mathrm{~min}^{-1}\right)$ é a constante cinética para adsorção de primeira ordem.

A Equação 7 apresenta uma relação linear entre $\log \left(\mathrm{q}_{\mathrm{e}}-\mathrm{q}_{\mathrm{t}}\right)$ e $\mathrm{t}$. Cálculos de regressão linear permitem obter os valores de $K_{1}$ e $q_{e}$. Estes valores foram calculados para a adsorção de $\mathrm{Cr}(\mathrm{VI})$ no compósito HT-Fe 500 e estão mostrados na Tabela 2.

$\mathrm{O}$ modelo linearizado de pseudossegunda ordem de $\mathrm{Ho}^{12}$ é representado pela Equação 8, onde $\mathrm{K}_{2}$ é a constante de segunda ordem $\left(\mathrm{g} \mathrm{mg}^{-1} \mathrm{~min}^{-1}\right)$ :

$$
\frac{\mathrm{t}}{\mathrm{q}_{\mathrm{t}}}=\frac{1}{\mathrm{qe}_{\mathrm{e}}} \mathrm{t}+\frac{1}{\mathrm{~K}_{2} \mathrm{qe}^{2}}
$$

Plotando-se $\mathrm{t} / \mathrm{q}_{\mathrm{t}}$ versus $\mathrm{t}$, os coeficientes angular e linear são, respectivamente, $1 / \mathrm{q}_{\mathrm{e}}$ e $1 /\left(\mathrm{K}_{2} \mathrm{q}_{\mathrm{e}}{ }^{2}\right)$. Os valores da constante de pseudossegunda ordem $\left(\mathrm{K}_{2}\right)$ e a capacidade de adsorção no equilíbrio $\left(\mathrm{q}_{\mathrm{e}}\right.$ ) foram obtidos a partir destes valores e constam na Tabela 2. A constante $\mathrm{K}_{2}$ foi utilizada ainda para calcular a razão de adsorção inicial h $\left(\mathrm{mg} \mathrm{g}^{-1} \mathrm{~min}^{-1}\right)$ para $\mathrm{t} \rightarrow 0$, a partir da Equação 9:36

$$
\mathrm{h}=\mathrm{K}_{2} \mathrm{qe}^{2}
$$


Tabela 2. Parâmetros cinéticos para a adsorção de Cr(VI) pelo compósito calcinado hidrotalcita-óxido de ferro calcinado (HT-Fe 500) (T = 298 K)

\begin{tabular}{|c|c|c|c|c|c|c|c|c|c|c|}
\hline \multirow[b]{2}{*}{$\mathrm{C}_{\mathrm{o}}\left(\mathrm{mg} \mathrm{L}^{-1}\right)$} & \multirow[b]{2}{*}{$\begin{array}{c}\text { qe exp } \\
\left(\mathrm{mg} \mathrm{g}^{-1}\right)\end{array}$} & \multicolumn{3}{|c|}{ Pseudoprimeira ordem } & \multicolumn{4}{|c|}{ Pseudossegunda ordem } & \multicolumn{2}{|c|}{ Dif. intrapartícula } \\
\hline & & $\mathrm{K}_{1}\left(\min ^{-1}\right)$ & qe $\left(\mathrm{mg} \mathrm{g}^{-1}\right)$ & $\mathrm{R}^{2}$ & $\begin{array}{c}\mathrm{K}_{2} \\
\left(\mathrm{~g} \mathrm{mg}^{-1} \min ^{-1}\right)\end{array}$ & qe $\left(\mathrm{mg} \mathrm{g}^{-1}\right)$ & $\begin{array}{c}\mathrm{h} \\
\left(\mathrm{mg} \mathrm{g}^{-1} \min ^{-1}\right)\end{array}$ & $\mathrm{R}^{2}$ & $\mathrm{~K}_{\mathrm{p}}$ & $\mathrm{R}^{2}$ \\
\hline 75 & 10,38 & $3,73 \times 10^{-3}$ & 8,81 & 0,927 & $8,17 \times 10^{-4}$ & 12,81 & 0,13 & 0,996 & 0,46 & 0,894 \\
\hline 100 & 15,84 & $3,50 \times 10^{-3}$ & 15,58 & 0,995 & $1,36 \times 10^{-4}$ & 21,94 & 0,066 & 0,991 & 1,36 & 0,988 \\
\hline 200 & 30,46 & $3,60 \times 10^{-3}$ & 31,16 & 0,995 & $9,23 \times 10^{-4}$ & 39,71 & 1,46 & 0,971 & 0,68 & 0,988 \\
\hline
\end{tabular}

O modelo de difusão intrapartícula ${ }^{37}$ é descrito através da Equação 10:

$$
\mathrm{q}_{\mathrm{t}}=\mathrm{K}_{\mathrm{p}} \mathrm{t}^{1 / 2}+\mathrm{C}
$$

onde $\mathrm{K}_{\mathrm{p}}$ é a constante de difusão intrapartícula $\left(\mathrm{mg} \mathrm{g}^{-1} \mathrm{~min}^{-0,5}\right)$ e C é a constante de adsorção que representa o coeficiente linear quando se plota o gráfico $\mathrm{q}_{\mathrm{t}}$ versus $\mathrm{t}^{1 / 2}$. Os resultados da aplicação deste modelo aos dados cinéticos da adsorção de Cr(VI) pelo HT-Fe 500 também são apresentados na Tabela 2 .

Embora os valores de $\mathrm{R}^{2}$ para os modelos de pesudoprimeira e pseudossegunda ordens sejam semelhantes e próximos da unidade $\left(\mathrm{R}^{2}>0,927\right)$, os resultados cinéticos na Tabela 2 mostram que os dados experimentais da adsorção de Cr(VI) pelo HT-Fe 500 se ajustam melhor ao modelo de pseudoprimeira ordem, pois os valores da capacidade de adsorção prevista por este modelo são muito próximos dos valores obtidos experimentalmente. A relação entre quantidade de $\mathrm{Cr}(\mathrm{VI})$ adsorvida versus tempo é mostrada na Figura 2S (material suplementar).

A partir da Figura $2 \mathrm{~S}$ verifica-se que para a concentração inicial de cromo(VI) igual a $75 \mathrm{mg} \mathrm{L}^{-1} \mathrm{o}$ tempo necessário de contato entre adsorvente e adsorvato para se atingir o equilíbrio é de aproximadamente 270 min. Já para as outras concentrações (100 e $200 \mathrm{mg} \mathrm{L}^{-1}$ ) avaliadas, o tempo foi aproximadamente o mesmo e igual a $420 \mathrm{~min}$.

$\mathrm{O}$ ajuste dos dados experimentais da adsorção de $\mathrm{Cr}(\mathrm{VI})$ pelo HT-Fe 500 ao modelo de pseudoprimeira ordem indica que a taxa de adsorção é diretamente proporcional à diferença entre as concentrações inicial e de equilíbrio do adsorvato e que o processo depende do número de íons metálicos presente em solução. O modelo de pseudoprimeira ordem, ao contrário do de pseudossegunda ordem, sugere que não há partilha ou troca de elétros entre metal e adsorvente, caracterizando o processo de adsorção entre adsorvente e adsorvato como uma fisiossorção. ${ }^{38}$

\section{Termodinâmica de adsorção}

Para se concluir se o processo é espontâneo ou não são necessárias considerações termodinâmicas de um processo de adsorção. ${ }^{12} \mathrm{Os}$ dados experimentais obtidos para a adsorção de $\mathrm{Cr}(\mathrm{VI})$ pelo HT-Fe 500 em diferentes temperaturas foram utilizados para o cálculo dos parâmetros termodinâmicos, tais como energia livre de Gibbs $\left(\Delta \mathrm{G}^{\circ}\right)$, variação de entalpia $\left(\Delta \mathrm{H}^{\circ}\right)$ e variação de entropia $\left(\Delta \mathrm{S}^{\circ}\right)$. A variação na energia livre de Gibbs foi calculada a partir da Equação 11, levando-se em consideração a constante de Langmuir, b, e também a Equação de van Hoff. ${ }^{38,39}$ Os resultados são mostrados na Tabela 3.

$$
\Delta \mathrm{G}^{\circ}=-\mathrm{RT} \ln (55,5 \mathrm{~b})
$$

onde, $\mathrm{R}$ é a constante dos gases $\left(8,314 \mathrm{~J} \mathrm{~mol}^{-1} \mathrm{~K}^{-1}\right)$ e T a temperatura (K).

A relação entre $\Delta \mathrm{G}^{\circ}, \Delta \mathrm{H}^{\circ}$ e $\Delta \mathrm{S}^{\circ}$ é expressa conforme a equação:

$$
\Delta \mathrm{G}^{\circ}=\Delta \mathrm{H}^{\circ}-\mathrm{T} \Delta \mathrm{S}^{\circ}
$$

A determinação de $\Delta \mathrm{H}^{\circ}$ e $\Delta \mathrm{S}^{\circ}$ é feita através da Equação 13:

$$
\ln (55,5 \mathrm{~b})=\frac{\Delta \mathrm{S}^{\circ}}{\mathrm{R}}-\frac{\Delta \mathrm{H}^{\circ}}{\mathrm{RT}}
$$

Plotando-se o gráfico de $\ln \left(55,5\right.$ b) versus $1 / \mathrm{T}$, os valores de $\Delta \mathrm{H}^{\circ}$ e $\Delta S^{\circ}$ foram determinados a partir dos coeficientes angular e linear, respectivamente, e apresentados na Tabela 3.

Tabela 3. Parâmetros termodinâmicos da adsorção de $\mathrm{Cr}(\mathrm{VI})$ pelo compósito calcinado HT-Fe 500

\begin{tabular}{ccccc}
\hline Temperatura & $\ln (55,5 \mathrm{~b})$ & $\begin{array}{c}\Delta \mathrm{G}^{\circ} / \\
\mathrm{KJ} \mathrm{mol}^{-1}\end{array}$ & $\begin{array}{c}\Delta \mathrm{H}^{\circ} / \\
\mathrm{KJ} \mathrm{mol}^{-1}\end{array}$ & $\begin{array}{c}\Delta \mathrm{S}^{\circ} / \\
\mathrm{KJ} \mathrm{mol}^{-1} \mathrm{~K}^{-1}\end{array}$ \\
\hline 298,15 & 1,97 & $-4,89$ & & \\
303,15 & 1,90 & $-4,79$ & & \\
308,15 & 1,81 & $-4,63$ & $-15,29$ & $-68,0$ \\
313,15 & 1,79 & $-4,67$ & & \\
318,15 & 1,54 & $-4,08$ & & \\
\hline
\end{tabular}

Os valores negativos de $\Delta \mathrm{G}^{\circ}$ apresentados na Tabela 3 confirmam a viabilidade do processo de adsorção e a natureza espontânea deste, o que potencializa o uso do adsorvente em processos convencionais de descontaminação de águas ou efluentes contendo cromo hexavalente. ${ }^{12}$ Já o valor negativo da variação de entalpia confirma a natureza exotérmica da adsorção e o valor negativo da variação de entropia padrão vem a confirmar o resultado apresentado pelo estudo cinético, ou seja, que o processo de remoção de $\mathrm{Cr}(\mathrm{VI})$ pelo compósito calcinado HT-Fe 500 se trata de uma adsorção física e não química. ${ }^{40} \mathrm{~A}$ magnitude do $\Delta \mathrm{H}^{\circ}$ também pode confirmar a natureza da adsorção, ou seja, entre 2,1 e $20,9 \mathrm{~kJ} \mathrm{~mol}^{-1}$ sugere que o processo de adsorção é físico e entre 20,9 e $418,4 \mathrm{~kJ} \mathrm{~mol}^{-1}$ é químico. ${ }^{37}$ No presente estudo, o valor de $\Delta \mathrm{H}^{\circ}$ é de $15,29 \mathrm{~kJ} \mathrm{~mol}^{-1}$, indicando que o processo de adsorção é físico. Devido à estrututura iônica lamelar das hidrotalcitas, as contribuições eletrostáticas são as principais forças envolvidas na adsorção física deste tipo de adsorvente. Os valores negativos de $\Delta \mathrm{G}^{\circ}$ indicam ainda uma alta preferência dos íons de Cr(VI) pelo adsorvente HT-Fe 500. ${ }^{12}$

Há também uma diminuição da desordem na interface sólidolíquido, o que é evidenciado pelo valor negativo de $\Delta \mathrm{S}^{\circ}$. Os valores negativos de $\Delta \mathrm{H}^{\circ}$ e $\Delta \mathrm{S}^{\circ}$ sugerem ainda que a entalpia contribuiu mais que a entropia para a obtenção de valores negativos de $\Delta \mathrm{G}^{\circ}$.

\section{CONCLUSÕES}

O presente estudo mostrou que o compósito magnético calcinado HT-Fe 500 pode ser usado como um adsorvente efetivo para remoção de $\mathrm{Cr}(\mathrm{VI})$ de soluções aquosas. Os resultados do estudo de pH mostraram que o processo de adsorção do $\mathrm{Cr}(\mathrm{VI})$ pelo HT-Fe 500 ocorre com um aumento da alcalinidade das soluções. $\mathrm{O}$ aumento de $\mathrm{pH}$ da solução favoreceu a remoção do $\mathrm{Cr}(\mathrm{VI})$, pois a espécie predominante em meio básico é o cromato $\left(\mathrm{CrO}_{4}^{2-}\right)$, uma espécie bivalente de $\mathrm{Cr}(\mathrm{VI})$ que apresenta alta interação eletrostática com o adsorvente. 
Os estudos cinéticos mostraram que os dados experimentais da adsorção de $\mathrm{Cr}(\mathrm{VI})$ no compósito calcinado HT-Fe 500 se ajustaram bem ao modelo de pseudoprimeira ordem, sugerindo que o processo de interação entre adsorvente e adsorvato ocorre por fisiossorção. O modelo de isoterma de três parâmetros de Langmuir-Freundlich apresentou o melhor ajuste para os dados experimentais no equilíbrio, indicando que a adsorção de $\mathrm{Cr}(\mathrm{VI})$ ocorre em monocamada na superfície heterogênea do adsorvente. As capacidades de adsorção calculadas a partir deste modelo foram 25,$93 ; 48,31 ; 39,78 ; 38,56 \mathrm{e}$ $40,17 \mathrm{mg} \mathrm{g}^{-1}$, respectivamente, para as temperaturas de $25,30,35,40$ e $45^{\circ} \mathrm{C}$. Já os dados termodinâmicos indicaram a espontaneidade, a natureza exotérmica e física da adsorção. A principal vantagem do adsorvente HT-Fe 500 em relação aos demais utilizados na remoção de íons metálicos de soluções aquosas é a sua facilidade de remoção de soluções aquosas por um simples processo magnético.

\section{MATERIAL SUPLEMENTAR}

O material suplementar, disponível gratuitamente em http://químicanova.sbq.org.br na forma de arquivo PDF, apresenta as seguintes figuras: comportamento do compósito HT-Fe 500 na presença de um imã (Figura 1S) e o efeito do tempo de contato na adsorção de $\mathrm{Cr}(\mathrm{VI})$ pelo compósito HT-Fe 500 (Figura 2S).

\section{AGRADECIMENTOS}

À FAPEMIG pelo apoio financeiro (Demanda Universal, processo n: APQ-00416-11) e ao Núcleo de Microscopia e Microanálise (NMM/UFV) pela realização das micrografias eletrônicas por varredura - MEV.

\section{REFERÊNCIAS}

1. Gómez, V.; Callao, M. P.; Trends Anal. Chem. 2006, 25, 1006.

2. Singh, A. K.; Gupta, V. K.; Gupta, B.; Anal. Chim. Acta 2007, 585, 171.

3. Basha, S.; Murthy, Z. V. P.; Jha, B.; Chem. Eng. J. 2008, 137, 480.

4. Moreno, R. A. S.; Gismera, M. J.; Sevilla, M. T.; Procopio, J. R.; Anal. Chim. Acta 2009, 634, 68

5. Malkoc, E.; Nuhoglu, Y.; Purif. Technol. 2007, 54, 291.

6. Owlad, M.; Aroua, M. K.; Daud, W. M. A. W.; Bioresour. Technol. 2010, 101,5098

7. Kotás, J.; Stasicka, Z.; Environ. Pollut. 2000, 107, 263.

8. Xiao, L.; Ma, W.; Han, M.; Cheng, Z.; J. Hazard. Mater. 2011, 186, 690.

9. Toledo, T. V.; Bellato, C. R.; do Rosário, R. H.; Marques Neto, J. de O.; Quim. Nova 2011, 34, 561.

10. Lazaridis, N. K.; Karapantsios, T. D.; Georgantas, D.; Water Res. 2003, $37,3023$.

11. Goh, K. H.; Lim, T. T.; Dong, Z.; Water Res. 2008, 42, 1343.

12. Hu, X.-J.; Wang, J.-S.; Liu, Y.-G.; Li, X.; Zeng, G.-M.; Bao, Z.-L.; Zeng, X.-X.; Chen, A.-W.; Long, F.; J. Hazard. Mater. 2011, 185, 306.
13. Denkbas, E. B.; Kiliçay, E.; Birlikseven, C.; Ozturk, E.; Water Res. 2002, 50, 225.

14. Reichle, W. T.; Solid States Ionics 1986, 22, 135

15. Pergher, S. B. C.; Oliveira, L. C. A.; Smaniotto, A.; Petkowicz, D. I.; Quim. Nova 2005, 28, 751.

16. Gillman, G. P.; Sci. Total Environ. 2006, 366, 926.

17. Rodrigues, J. C.; Dissertação de Mestrado, Universidade Federal do Rio Grande do Sul, Brasil, 2007.

18. Setz, L. F. G.; Tese de Doutorado, Instituto de Pesquisas Energéticas e Nucleares, Brasil, 2009.

19. Maia, A. S.; Osorio, V. K. L.; Quim. Nova 2003, 26, 595.

20. Cornell, R. M.; Schwertmann, U.; The Iron Oxides, $3^{\text {rd }}$ ed., WeinheimVHC: New York, 2003.

21. Gonçalves, M.; Oliveira, L. C. A.; Guerreiro, M. C.; Quim. Nova 2008, 31, 518.

22. Yang, L.; Shahrivari, Z.; Liu, P. K. T.; Sahimi, M.; Tsotsis, T. T.; Ind. Eng. Chem. Res. 2005, 44, 6804.

23. Conceição, L.; Pergher, S. B. C.; Moro, C. C.; Oliveira, L. C. A.; Quim. Nova 2007, 30, 1077.

24. Bastiani, R.; Dissertação de Mestrado, Universidade Federal do Rio de Janeiro, Brasil, 2001.

25. Goswamee, R. L.; Sengupta, P.; Bhattacharyya, K. G.; Dutta, D. K.; Appl. Clay Sci. 1998, 13, 21.

26. Aksu, Z.; Process Biochem. 2002, 38, 89.

27. Li, Y. H.; Di, Z.; Ding, J.; Wu, D.; Luan, Z.; Zhu, Y.; Water Res. 2005, 39,605 .

28. Guilarduci, V. V. S.; Mesquita, J. P.; Martelli, P. B.; Gorgulho, H. F.; Quim. Nova 2006, 29, 1226.

29. Brinques, G. B.; Dissertação de Mestrado, Universidade Federal do Rio Grande do Sul, Brasil, 2005.

30. Santos, E. B.; Dissertação de Mestrado, Universidade Federal de Sergipe, Brasil, 2008.

31. Álvarez-Ayuso, E.; Nugteren, H. W.; Water Res. 2005, 39, 2535.

32. Das, N. N.; Konar, J.; Mohanta, M. K.; Srivastava, S. C.; J. Colloid Interface Sci. 2004, 270, 1.

33. Manju, G. N.; Gigi, M. C.; Anirudhan, T. S.; Indian J. Chem. Tech. 1999, $6,134$.

34. Febrianto, J.; Kosasih, A. N.; Sunarso, J.; Ju, Y. -H.; Indraswati, N.; Ismadji, S.; J. Hazard. Mater. 2009, 162, 616.

35. Tseng, R.-L.; Wu, F.-C.; Juang, R.-S.; J. Taiwan Inst. Chem. Eng. 2010, 41,661 .

36. Djeribi, R.; Hamdaoui, O.; Desalination 2008, 225, 95.

37. Venugopal, V.; Mohanty, K.; Chem. Eng. J. 2011, 174, 151.

38. Khambhaty, Y.; Mody, K.; Basha, S.; Jha, B.; Chem. Eng. J. 2009, 145, 489.

39. Albadarin, A. B.; Mangwandi, C.; Al-Muhtaseb, A. H.; Walker, G. M.; Allen, S. J.; Ahmad, M. N. M.; Chem. Eng. J. 2012, 179, 193.

40. Fernandes, F. L.; Tese de Doutorado, Universidade Federal da Paraíba, Brasil, 2008. 